\title{
Study protocol: associations between dietary patterns, cognitive function and metabolic syndrome in older adults - a cross-sectional study
}

Karen D. Mumme ${ }^{1}$, Pamela R. von Hurst ${ }^{1}$, Cathryn A. Conlon ${ }^{1}$, Beatrix Jones², Crystal F. Haskell-Ramsay ${ }^{3}$, Welma Stonehouse ${ }^{4}$, Anne-Louise M. Heath ${ }^{5}$, Jane Coad ${ }^{6}$ and Kathryn L. Beck ${ }^{1 *}$ (D)

\begin{abstract}
Background: Loss of cognitive function is a significant issue as the world's population ages. Preserving cognitive function maintains independence in older adults bringing major societal and financial benefits. Lifestyle factors such as diet are modifiable risk factors, which may help preserve cognitive function.

Most nutrition research aimed at preserving cognitive function and metabolic health has focussed on individual nutrients and foods, not allowing for food combinations and interactions. A dietary pattern approach considers the entire diet including its complexity. Previous research investigating dietary patterns and cognitive function has not always considered relevant covariates such as physical activity and the Apolipoprotein E genotype, which are known to have associations with cognitive function.

The aim of the REACH (Researching Eating, Activity and Cognitive Health) study is to investigate associations between dietary patterns, cognitive function and metabolic syndrome, accounting for a range of covariates.

Methods: This cross-sectional study design will recruit older, community-living adults (65-74 years) from Auckland, New Zealand. Dietary data will be collected via a 109-item food frequency questionnaire validated using a 4-day food record. Cognitive function will be assessed using the Montreal Cognitive Assessment (paper based) and the Computerised Mental Performance Assessment System (COMPASS) - a testing suite covering six domains. Additional data will include genetic (Apolipoprotein E ع4) and biochemical markers (fasting glucose, HbA1c, lipids profile), anthropometric measurements (weight, height, waist and hip circumference, body composition using dual X-ray absorptiometry), blood pressure, physical activity (International Physical Activity Questionnaire - short form) and health and demographics (questionnaire).

Dietary patterns will be derived by principal component analysis. Associations between cognitive function and dietary patterns will be examined using multiple regression analysis. Covariates and interaction factors will include age, education, socio-economic status, physical activity, Apolipoprotein E $\varepsilon 4$ genotype, family history of dementia or cognitive impairment, and lifestyle factors. Differences between participants with and without metabolic syndrome will also be examined.

(Continued on next page)
\end{abstract}

\footnotetext{
* Correspondence: k.l.beck@massey.ac.nz

${ }^{1}$ College of Health, Massey University, Private Bag 102904, Auckland 0745,

New Zealand

Full list of author information is available at the end of the article
}

(c) The Author(s). 2019 Open Access This article is distributed under the terms of the Creative Commons Attribution 4.0 International License (http://creativecommons.org/licenses/by/4.0/), which permits unrestricted use, distribution, and reproduction in any medium, provided you give appropriate credit to the original author(s) and the source, provide a link to the Creative Commons license, and indicate if changes were made. The Creative Commons Public Domain Dedication waiver (http://creativecommons.org/publicdomain/zero/1.0/) applies to the data made available in this article, unless otherwise stated. 
(Continued from previous page)

Discussion: This study will bring new knowledge regarding associations between dietary patterns and cognitive function and metabolic health in older adults living in New Zealand. This is important for developing nutrition related recommendations to help older adults maintain cognitive function.

Keywords: Dietary patterns, Cognitive decline, Cognition, Metabolic syndrome, Older adults, Principal component analysis, New Zealand

\section{Background}

The global population aged over 60 years has doubled in the last 30 years and is expected to double again by 2050 [1]. Independence and quality of life are valued by older adults [2] but these attributes are threatened by poor health in later years [3]. Delaying entry of older adults into aged care residential facilities brings substantial financial benefits to individuals and governments [4]. A key predictor for admission into residential care is impaired cognitive function or dementia [5]. Loss of cognitive function takes away independence, a healthy fulfilling life in final years and can significantly affect family members [6]. Hence, there is a case for preserving cognitive function for as long as possible.

Age-related cognitive decline, mild cognitive impairment and dementia are progressive with limited pharmacological and non-pharmacological treatments available [7]. Without a cure, researchers should strive to discover ways to prevent or slow cognitive decline and the onset of dementia through lifestyle and dietary changes [6].

Age and genetic factors are the most significant risk factors for developing dementia [6]. One specific genetic factor, Apolipoprotein E \&4 (ApoE ع4) not only increases the risk for developing Alzheimer's Disease [8] and vascular dementia [9] but also puts healthy individuals at risk of a faster cognitive decline as they age [10]. Age and genotype cannot be altered but there are some modifiable risk factors [11] which may slow cognitive decline.

Modifiable risk factors include physical inactivity, smoking, hypertension, obesity, type 2 diabetes mellitus and low educational attainment [11]. Three of these risk factors (hypertension, obesity and diabetes) are components of metabolic syndrome, which further suggests the presence of a "metabolic-cognitive syndrome" via their similar underlying mechanisms through vascular changes [12]. The relevance of lifestyle and diet in cognitive decline and metabolic syndrome is well-recognised [13] and lifestyle and diet is a starting point in the prevention, delay or reduction of the impact of cognitive decline.

Foods and nutrients that may protect against the loss of cognitive function have been identified. These include fruits and vegetables [14-16], fish [15, 16], mono- and poly-unsaturated fats $[15,16]$ and antioxidants $[17,18]$. In contrast, nutrients such as refined carbohydrates and saturated fat may impair cognitive function $[15,19]$.
Studies of food groups and macro- and micro-nutrients make a valued contribution to nutritional science. However, people consume a diet consisting of many foods, and single nutrient or food group studies may have outcomes confounded by the overall complexity of the diet. The food combinations within a diet can act synergistically, for example altering the bioavailability of nutrients within the body. These changes are not considered in studies of individual food groups or nutrients alone [20]. The study of the combinations and complexities of dietary intake is a more recent development and dietary pattern analysis acknowledges the complexities of food combinations and their associated biochemical interactions as important [21].

Dietary patterns are derived through two processes using dietary data. The first process uses theoretically derived (a priori) dietary patterns based on current nutritional knowledge. A dietary index is created, ranking study participants' adherence to dietary guidelines (e.g. US dietary guidelines) or a dietary pattern (e.g. the Mediterranean diet index) [20]. Several studies show better cognitive function in older adults if their normal dietary intake follow specific features of a diet, for example the Mediterranean diet with its high intake of vegetables, fruits, fish, nuts, cereals and olive oil [16] or the Dietary Approach to Stop Hypertension (DASH) diet, high in vegetables and dairy with a low consumption of saturated fats and sodium [15]. Additionally, the Mediterranean diet, DASH and a Nordic diet, based on Nordic nutrition recommendations, may also have benefits with regards to metabolic syndrome [22].

The second process is an empirically derived (a posteriori) dietary pattern. The statistical method of principal component analysis allows measurements on many dietary components to be reduced to measures on a few different dietary patterns [20]. This method enables a better understanding of eating behaviours and a unique view of dietary intake [23] within a population setting. With regards to the older adult, positive associations between empirically derived dietary patterns and cognitive function have been recognised in 'healthy/prudent' [24-26] or 'carotenoid-rich' [27] dietary patterns in primarily European or North American populations and 'plant food and fish' [28], 'vegetables-fruits' [29] and 'snack-drinks-milk products' [29] dietary patterns in Asian populations. 
Conversely, negative associations have been noted in cognitive function where a 'Western' [25] dietary pattern was consumed. In some instances, no associations were found between cognitive function and 'traditional' [26],'meat-fish [29], 'health aware' [30] or 'sweet foods' [30] dietary patterns.

Associations can be attenuated when important covariates are included in the statistical analysis suggesting that the covariate has an effect on the outcome. For example, negative associations between cognitive function and 'high red meat' [31], 'high butter' [31], 'processed food' [32] and 'traditional' [30] dietary patterns were weakened when the ApoE ع4 genotype [31], education [32] or childhood IQ [30] were considered in the analysis. Likewise, a positive association was weakened in a 'whole food' and 'Mediterranean style' dietary pattern with education [32] or childhood IQ [30] as covariates. Consideration of covariates is critical; without it some studies may not be as robust and lead to misinformed conclusions. One of these covariates is the ApoE \&4 genotype. This genotype has been considered in recent research. Analyses show associations are still apparent between dietary patterns and cognitive function when ApoE $\varepsilon 4$ is adjusted for [33-35], though in older studies this covariate was disregarded [24-26, 29, 32, 36].

A 'dietary pattern' is population specific and dietary patterns have been studied in the New Zealand context [37-42]. However, no studies have investigated dietary patterns exclusively in an older New Zealand population. Therefore, the objectives of the REACH (Researching Eating, Activity and Cognitive Health) study are to investigate associations between dietary patterns and cognitive function in older adults within the New Zealand population. A secondary objective is to investigate associations between dietary patterns and metabolic syndrome.

\section{Methods}

\section{Study design and participants}

The participants in this cross-sectional study will be men and women aged 65-74 years, living independently in Auckland, New Zealand and proficient in English. Participants will be excluded if they are colour blind (due to the computerised cognitive testing requiring colour recognition); have a diagnosis of dementia or any of the following conditions which may impair cognitive function: stroke, traumatic head or brain injury, a neurological or psychiatric condition; or if they are taking medication which may influence their cognitive function. Another exclusion criterion is any event in the last 2 years which had a substantial impact on dietary intake and cognitive function, for example, death or illness of a family member.
A sample size of 350 participants is required to see a medium size effect (Pearson correlation 0.3) with $80 \%$ power for the main outcome of a linear association between the cognitive scales and dietary patterns. This sample size is similar to other studies investigating cognitive function and dietary patterns [36]. Data will be collected from 360 participants to allow for missing or incomplete data.

Recruitment and data collection will commence in 2018 and is expected to take 12 months. Participants will be required to attend the Human Nutrition Research Unit, Massey University, Auckland, New Zealand on one occasion. Informed consent forms will be completed at the research facility prior to data collection. The research day involves collecting health and demographic data, blood pressure, anthropometric and physical activity data as per Table 1. A fasted blood sample will be taken prior to a standardised breakfast. Two cognitive assessments will be completed after breakfast. An online food frequency questionnaire (FFQ) and a food diary information video complete the session.

Funding is provided by the Health Research Council of New Zealand, Grant 17/566. Ethical approval has been granted by Massey University Human Ethics Committee: Southern A, Application 17/69.

\section{Recruitment and screening}

Participants will be recruited throughout the wider Auckland region via several channels including: the Human Nutrition Research Unit, Massey University participant database; media, including radio interviews and press releases from Massey University; posters and flyers at local libraries, community centres, recreation centres, sports and hobby clubs, Citizens Advice Bureaus, retirement villages and second hand shops; inclusion in relevant newsletters, e.g. Age Concern New Zealand; and online promotion on appropriate social media pages e.g. GrownUps, Office for Seniors Facebook page. The REACH study will have a website where potential participants will be directed for further information and to register interest [48]. Only one person per household will be eligible to participate in the study. Participants expressing an interest in the study will be provided with an information sheet and undergo a screening interview [48], via telephone, to ensure inclusion criteria are met.

\section{Health, demographic, and physical activity data}

Health, demographics, lifestyle and physical activity information will be collected through written questionnaires in person (Table 1). Data quality will be ensured by checking questionnaires for completeness and sensibility. If further clarification is required, it will be done immediately or within a few days by 
Table 1 Outcome measures and testing methods for data collection

\begin{tabular}{|c|c|}
\hline Variables & Methods \\
\hline \multicolumn{2}{|l|}{ Questionnaires } \\
\hline Health and demographics & $\begin{array}{l}\text { Written questionnaire developed by } \\
\text { the researchers - questions regarding: } \\
\text { socio-demographic, health, lifestyle and } \\
\text { dietary factors. }\end{array}$ \\
\hline Physical Activity & $\begin{array}{l}\text { International Physical Activity } \\
\text { Questionnaire - short form [43] }\end{array}$ \\
\hline \multicolumn{2}{|l|}{ Anthropometry } \\
\hline $\begin{array}{l}\text { Height, weight, waist and } \\
\text { hip circumference }\end{array}$ & $\begin{array}{l}\text { ISAK }{ }^{\mathrm{b}} \text { anthropometry methods } \\
\text { [44] - stadiometer, Tanita Electronic } \\
\text { Scales, Lufkin W600 PM flexible steel tape }\end{array}$ \\
\hline Muscle mass and fat mass & $\begin{array}{l}\text { Dual-emission X-ray absorptiometry, } \\
\text { Hologic, Discovery QDR series }\end{array}$ \\
\hline \multicolumn{2}{|l|}{ Blood Analysis } \\
\hline Fasting blood glucose & HemoCue Glucose 201RT \\
\hline $\mathrm{HbA} 1 \mathrm{c}$ & Cobas b 101 system [45] \\
\hline $\begin{array}{l}\text { Lipid Profile (total cholesterol, } \\
\text { triglycerides, } \mathrm{HDL}-\mathrm{C}, \mathrm{LDL}-\mathrm{C}^{\mathrm{a}} \text { ) }\end{array}$ & Cobas b 101 system [45] \\
\hline Apolipoprotein E ع4 & $\begin{array}{l}\text { Polymerase chain reaction amplification } \\
\text { and direct nucleotide sequence analysis }\end{array}$ \\
\hline \multicolumn{2}{|l|}{ Clinical } \\
\hline Blood pressure & $\begin{array}{l}\text { Digital Automatic Blood Pressure } \\
\text { Monitor, Omron HEM-907 }\end{array}$ \\
\hline \multicolumn{2}{|l|}{ Dietary intake } \\
\hline $\begin{array}{l}\text { Food Frequency } \\
\text { Questionnaire }\end{array}$ & $\begin{array}{l}\text { Via Survey Monkey - adapted from } \\
\text { Beck et al. [46] }\end{array}$ \\
\hline Estimated 4-day food diary & Paper form \\
\hline \multicolumn{2}{|l|}{ Cognitive tests } \\
\hline Global cognitive function & $\begin{array}{l}\text { Montreal Cognitive Assessment } \\
(\text { MoCA) [47] }\end{array}$ \\
\hline Multiple cognitive domains & $\begin{array}{l}\text { Computerised Mental Performance } \\
\text { Assessment System } \\
\text { (COMPASS-Northumbria University, } \\
\text { Newcastle upon Tyne, UK) refer Table } 2\end{array}$ \\
\hline
\end{tabular}

acalculated

${ }^{b}$ International Society for the Advancement of Kinanthropometry

phone or email. Socio-demographic information includes age, gender, ethnicity, marital status, education, household income, work history, living situation and food security. Health information will include past and current disease (acute and chronic), medication, family history of dementia or cognitive impairment, vision, hearing, dental health and mobility issues. Lifestyle information will include smoking history, alcohol intake, substance abuse, supplement use and changes in physical activity. Dietary information will include changes in diet over past 10 years and possible factors behind these changes e.g., dentition, health concerns, disposable income, appetite. An Index of Multiple Deprivation will be calculated from the residential address of participants [49].

\section{Anthropometric data and blood pressure}

Anthropometry, including body fat percentage, BMI, hip and waist circumference, and blood pressure will be measured as in Table 1.

Blood pressure will be measured twice. Participants will rest quietly (seated) for $5 \mathrm{~min}$ before the first measurement is taken. There will be a 1-min rest period between measurements. If either systolic or diastolic measurements differ by more than $5 \mathrm{mmHg}$ between measurements, a third measurement will be taken.

\section{Blood sampling, processing, analysis and genotyping}

For consistency, all participants will be fasted (except water) from $2200 \mathrm{~h}$ the night prior to their visit. Between 0700 and 0900 at the research facility, a qualified phlebotomist will draw fasted blood samples in the following order: $10 \mathrm{ml}$ BD Vacutainer ${ }^{\circ}$ Plus (cat 367895), $10 \mathrm{ml} \mathrm{BD}$ Vacutainer $^{\oplus}$ K2EDTA (cat 367525) and $6 \mathrm{ml} \mathrm{BD} \mathrm{Vacutai-}$ ner ${ }^{\oplus}$ K2EDTA (cat 367873). The $10 \mathrm{ml}$ BD Vacutainer ${ }^{\circ}$ Plus will be maintained at an ambient temperature for $30 \mathrm{~min}$ to allow clotting, then will be centrifuged with the $10 \mathrm{ml} \mathrm{BD} \mathrm{Vacutainer}{ }^{\ominus}$ K2EDTA (Heraeus Labofuge $400 \mathrm{R})$ for $15 \mathrm{~min}$ at $1547 \mathrm{~g}$-force $(3500 \mathrm{rpm})$ at $4{ }^{\circ} \mathrm{C}$. The resulting serum, from the $10 \mathrm{ml}$ BD Vacutainer ${ }^{\oplus}$ Plus, will be aliquoted into four Eppendorf tubes which will be stored for further measures. The resulting plasma, from the $10 \mathrm{ml}$ BD Vacutainer K2EDTA, will be aliquoted into six Eppendorf tubes and stored for further research. The $6 \mathrm{ml}$ EDTA vacutainer will be placed on ice and tested for blood glucose, HbA1c and a lipid profile using point-of-care equipment (Table 1). The remaining blood $(\sim 5 \mathrm{ml})$ will be stored for ApoE $\varepsilon 4$ analysis (Table 1) by an accredited laboratory, and two Eppendorf tubes $(500 \mu \mathrm{l})$ will be kept for backup. All samples will be stored at $-80^{\circ} \mathrm{C}$.

\section{Assessment of dietary intake}

The study spans a 10-month period from autumn to summer. Dietary data will be collected using two methods. First, a self-administered 109-item FFQ will collect data, covering the previous month, via an online survey on two occasions: at the research facility (FFQ1) and 1 month later (at home) (FFQ2) to assess reproducibility. The FFQ is adapted from a validated New Zealand FFQ aimed at assessing iron related dietary patterns in young women [46]. Changes included the addition of serving sizes; combining some food groups to shorten the questionnaire; the addition of foods not included in the initial nutrient specific FFQ e.g. confectionary; as well as extra questions for added clarification e.g. type of milk or oils used. The FFQ was further cross-checked with the New Zealand Women's Food Frequency Questionnaire $[50,51]$ to ensure all relevant food groups were included. Ten individuals in the study age range 
pre-tested the FFQ for understanding and readability. The completed FFQ will be entered into Foodworks 9 (Xyris Software, 2017), which uses the New Zealand FOODfiles $^{(\mathrm{ma})} 2016$ [52] food composition database. After data entry and inspection, any necessary adjustments will be made e.g. Goldberg Cut-off for energy intake [53].

Second, an estimated 4-day food diary will be collected from a subset of participants to validate the modified FFQ for food and dietary patterns. Participants will complete the 4-day food diary within 1 month of the study visit. The 4-day food diary covers four consecutive days including at least one weekend day. Prior to completing the 4-day food diary, participants will view an instructional video which explains the need to record all foods and beverages consumed including type, brands, and cooking methods. Participants will be taught how to estimate quantities using pictures [54], household measures, and measuring scales. The 4-day food diary will be processed by four trained nutritionists using Foodworks 9 (Xyris Software, 2017). A register of common food items will be kept ensuring consistency in data entry among the four nutritionists. Additionally, every tenth food record entered will be audited for accuracy and consistency.

\section{Cognitive assessment}

Cognitive testing will be carried out after a standardised breakfast to minimise any effects food may have on cognition. The cognitive testing will be undertaken in two parts. First, a trained examiner will administer the Montreal Cognitive Assessment (MoCA) on a one to one basis. MoCA is a clinically available and validated [47] tool. It takes $10 \mathrm{~min}$, assesses global cognitive function, short-term memory, visuospatial and executive function, attention, language and orientation, concentration and working memory [47]. MoCA will be used to provide comparisons with other studies, and as a descriptor.

The second part will be the Computerised Mental Performance Assessment System (COMPASS-Northumbria University, Newcastle upon Tyne, UK). This software platform presents tasks on desktop computers using a variety of methods to collect responses: mouse and cursor, a four-button coloured response pad, and pen and paper for word recall. This broad battery of tests assesses all cognitive domains (Table 2) and is sensitive to normal age-related effects and dietary factors $[55,56]$.

The reaction time for correct responses will be assessed and participants will be assigned a composite score for global cognitive function and for each cognitive domain.

Cognitive testing will be undertaken in a cognitive suite controlled for environmental factors e.g. noise and temperature. The tests will be taken at a similar time of day and participants will be instructed to avoid undue stress, alcohol, recreational drugs and physical activity
Table 2 The COMPASS ${ }^{a}$ battery of assessments

\begin{tabular}{|c|c|c|}
\hline $\begin{array}{l}\text { Cognitive } \\
\text { Domain }\end{array}$ & Definition & Test \\
\hline Mood & Measurement of subjective feelings & $\begin{array}{l}\text { Bond and Lader } \\
\text { Mood Scales }\end{array}$ \\
\hline \multirow[t]{3}{*}{$\begin{array}{l}\text { Attention and } \\
\text { vigilance }\end{array}$} & \multirow{2}{*}{$\begin{array}{l}\text { Attention - ability to concentrate on } \\
\text { selected aspects of the environment } \\
\text { while ignoring other stimuli }\end{array}$} & $\begin{array}{l}\text { Simple reaction } \\
\text { time }\end{array}$ \\
\hline & & $\begin{array}{l}\text { Choice reaction } \\
\text { time }\end{array}$ \\
\hline & $\begin{array}{l}\text { Vigilance - ability to maintain } \\
\text { attention and alertness over time }\end{array}$ & $\begin{array}{l}\text { Digit vigilance } \\
\text { task }\end{array}$ \\
\hline $\begin{array}{l}\text { Executive } \\
\text { function }\end{array}$ & $\begin{array}{l}\text { Co-ordination of cognitive responses } \\
\text { - sub-serves planning, initiating and } \\
\text { inhibiting actions, cognitive flexibility, } \\
\text { abstract thinking and rule acquisition }\end{array}$ & Stroop test \\
\hline \multirow[t]{3}{*}{$\begin{array}{l}\text { Episodic } \\
\text { memory }\end{array}$} & \multirow[t]{3}{*}{$\begin{array}{l}\text { Ability to retain memories that can } \\
\text { be consciously recorded e.g. facts, } \\
\text { items, events, faces }\end{array}$} & $\begin{array}{l}\text { Immediate and } \\
\text { delayed word } \\
\text { recall }\end{array}$ \\
\hline & & $\begin{array}{l}\text { Delayed word } \\
\text { recognition }\end{array}$ \\
\hline & & $\begin{array}{l}\text { Delayed picture } \\
\text { recognition }\end{array}$ \\
\hline $\begin{array}{l}\text { Working } \\
\text { memory }\end{array}$ & $\begin{array}{l}\text { Ability to hold information in mind } \\
\text { while carrying out more complex } \\
\text { cognitive processes }\end{array}$ & Corsi blocks \\
\hline \multirow[t]{2}{*}{$\begin{array}{l}\text { Location } \\
\text { learning }\end{array}$} & \multirow[t]{2}{*}{ Assesses visuo-spatial memory } & $\begin{array}{l}\text { Computerised } \\
\text { location learning }\end{array}$ \\
\hline & & $\begin{array}{l}\text { Computerised } \\
\text { location recall }\end{array}$ \\
\hline
\end{tabular}

${ }^{a}$ Computerised Mental Performance Assessment System (COMPASS-

Northumbria University, Newcastle upon Tyne, UK)

that is not routine prior to their appointment. Testing will take approximately $1 \mathrm{~h}$. The first $15 \mathrm{~min}$ will be training. The investigator will verbally describe the tasks, the format of the testing, and use of the response pad, and will answer any questions. The practice tests will be shorter and easier versions of the actual test. Participants will have a 5-min break before actual assessment is undertaken. The investigator will ensure any new computer users are comfortable with using a mouse before testing starts.

\section{Metabolic syndrome}

To determine the incidence of metabolic syndrome the criteria recommended by the American Heart Association/National Health, Lung and Blood Institute Scientific Statement will be followed [57]. Metabolic syndrome will be considered to exist where three of the following five criteria are met or medication is used to treat: waist circumference $\geq 88 \mathrm{~cm}$ for women and $\geq 102 \mathrm{~cm}$ for men; a triglyceride level of $\geq 1.7 \mathrm{mmol} / \mathrm{L}$; HDL cholesterol level of $<1.03 \mathrm{mmol} / \mathrm{L}$ in men or $<1.3 \mathrm{mmol} / \mathrm{L}$ in women; blood pressure $\geq 130 / 85 \mathrm{mmHg}$; fasting blood glucose $\geq 5.6$ $\mathrm{mmol} / \mathrm{L}$. 


\section{Provision of results to participants}

Feedback to participants after assessment will include anthropometric measurements (height, weight, BMI, waist and hip circumference, body fat \%), blood pressure and blood results (HbA1c, fasting blood glucose, lipid profile). A registered (NZ Medical Council) general practitioner will review all biochemical results prior to communicating them to participants. On completion of the study, participants will receive a report summarising the main findings of the REACH study.

\section{Statistical analysis}

Statistical analysis will be performed using R [58]. Participant data will be described using mean (95\% confidence intervals) for normally distributed data, median (25, 75 percentile) for non-normally distributed data, or frequency summary statistics for categorical data. The Shapiro-Wilk test and normality plot will evaluate the normality of distributions.

Dietary patterns will be identified using rotated principal component analysis, a statistical technique to reduce data and produce patterns based on the correlations between food groups [23]. The FFQ food group items will be further collapsed based on other studies investigating dietary patterns $[40,41]$. Three separate analyses will be performed: FFQ1 to determine dietary patterns, the 4-day food diary to assess validity of the dietary patterns identified in FFQ1, and FFQ2 vs FFQ1 to assess reproducibility. Orthogonal varimax rotation will be used to facilitate interpretability of components. The number of components retained will be based on the scree plot, eigenvalue $(>1)$, and interpretability of the dietary pattern. The Kaiser-Meyer-Olkin measure of sampling adequacy and Bartlett's Test $P$ values (to determine the presence of relationships between variables in the factor analysis) will be examined [59]. Labelling of dietary patterns will be based on the interpretation of foods with high factor loadings for each dietary pattern [60].

Multivariate multiple regression analysis [61] will be used to determine the association between dietary patterns and various domains of cognitive function (dependent variable) while considering confounding factors. Possible confounding and interaction factors include: age, gender, ethnicity, education, English as a second language, presence of chronic disease (including metabolic syndrome), socio-economic status, physical activity, body mass index, ApoE $\varepsilon 4$ genotype, family history of dementia, smoking, alcohol intake and past dietary intake.

Further analysis will compare dietary patterns of participants with and without metabolic syndrome using multiple logistic regression analysis.

\section{Discussion}

Age-related cognitive decline is a continuum of natural cognitive changes that may progress into mild cognitive impairment or dementia. Rates of decline differ within a population. Delaying the onset of cognitive decline is the optimal strategy but where there is an earlier on-set the ability to slow the decline is important. Delaying or slowing the decline may be abetted by modifiable lifestyle factors e.g. diet, physical activity. Therefore, an in-depth understanding of the diet (beyond an isolated food and nutrient approach) of older adults is warranted. The REACH study will broaden the current knowledge on dietary patterns and their associations with cognitive function and metabolic health in older adults in a New Zealand context.

Despite the cross-sectional design having limitations e.g. causality cannot be inferred, the study design has several strengths. The study recognises dietary patterns as a measure of the diet and acknowledges the whole diet is greater than the sum of its parts. Extra strengths come from the collection of data on the ApoE $\varepsilon 4$ allele, physical activity levels and other known covariates to ensure that any association found is likely to be due to the dietary patterns identified. Additionally, the COMPASS battery of cognitive tests is administered on the computer minimising potential bias associated with administration of these tests by a researcher. The scoring of cognition and the assessment of dietary patterns will be done independently to minimise bias. While there is always bias associated with the type of participant attracted to such studies, the bias will be minimised by using a range of recruiting methods across the wider Auckland region to ensure a range of participant demographics are captured. Furthermore, the size of the study population ensures there is adequate power to detect a meaningful result.

The REACH study is the first to examine dietary pattern associations in older New Zealand adults. This first step paves the way for developing recommendations for New Zealanders to maintain cognitive function, metabolic health and ultimately quality of life as they age. Additionally, it will provide a valuable base for hypothesis generation for future longitudinal studies and / or randomised controlled trials.

\section{Abbreviations \\ ApoE ع4: Apolipoprotein E ع4; COMPASS: Computerised Mental Performance Assessment System; DASH: Dietary Approach to Stop Hypertension; FFQ: Food Frequency Questionnaire; FFQ1: First Food Frequency Questionnaire; FFQ2: Second Food Frequency Questionnaire; ISAK: International Society for the Advancement of Kinanthropometry; MoCA: Montreal Cognitive Assessment}

\section{Acknowledgements}

We thank the REACH team, including Owen Mugridge and Cassie Slade for managing recruitment and Cheryl Gammon for advice on inclusion/ exclusion criteria regarding use of medication affecting cognitive function.

\section{Funding}

Funding is provided by a Health Research Council of New Zealand Emerging Researcher Grant 17/566 - Beck: Optimising cognitive function: the role of 
dietary and lifestyle patterns. The funders have no role in the design of the study; collection, analysis and interpretation of the data; writing manuscripts or publishing results.

\section{Availability of data and materials}

Data not yet available.

\section{Authors' contributions}

$\mathrm{KB}, \mathrm{PVH}, \mathrm{CC}, \mathrm{BJ}, \mathrm{CHR}, \mathrm{WS}, \mathrm{ALH}, \mathrm{JC}$ conceived and designed the study and acquired funding; $\mathrm{KB}$ acquired ethics approval; $\mathrm{KB}, \mathrm{PvH}, \mathrm{CC}, \mathrm{BJ}, \mathrm{CHR}$, WS, ALH, $\mathrm{KM}$ designed the food frequency questionnaires; $\mathrm{KB}, \mathrm{PvH}, \mathrm{CC}, \mathrm{BJ}, \mathrm{CHR}, \mathrm{WS}$, $\mathrm{ALH}$, JC designed the questionnaires; $\mathrm{KB}$, PvH, CC, KM will collect data; $\mathrm{KB}$, KM will coordinate recruitment, manage participant and data collection; KM drafted and wrote the manuscript; BJ advised on statistical analysis. All authors read and approved the final manuscript.

\section{Ethics approval and consent to participate}

Ethical approval was granted by Massey University Human Ethics Committee: Southern A, Application 17/69.

All participants will provide written informed consent, prior to commencement of data collection at the research facility.

\section{Consent for publication}

Not applicable.

\section{Competing interests}

The authors declare that they have no competing interests.

\section{Publisher's Note}

Springer Nature remains neutral with regard to jurisdictional claims in published maps and institutional affiliations.

\section{Author details \\ ${ }^{1}$ College of Health, Massey University, Private Bag 102904, Auckland 0745 , New Zealand. ${ }^{2}$ Department of Statistics, University of Auckland, Private Bag 92019, Auckland 1142, New Zealand. ${ }^{3}$ Department of Psychology, Northumbria University, Newcastle-upon-Tyne NE1 8ST, UK. ${ }^{4}$ Health and Biosecurity Business Unit, Commonwealth Scientific Industrial Research Organisation, PO Box 11060, Adelaide, SA 5001, Australia. ${ }^{5}$ Department of Human Nutrition, University of Otago, PO Box 56, Dunedin 9054, New Zealand. ${ }^{6}$ College of Sciences, Massey University, Private Bag 11222 Palmerston North 4442, New Zealand.}

Received: 17 February 2019 Accepted: 25 April 2019

Published online: 10 May 2019

\section{References}

1. United Nations, Department of Economic and Social Affairs, Population division. World population ageing 2017 - highlights (ST/ESA/SER.A/408). 2017. http://www.un.org/en/development/desa/population/publications/ pdf/ageing/WPA2017_Highlights.pdf, Accessed 28 Sept 2018.

2. Blazer DG, Yaffe K, Karlawish J. Cognitive aging: a report from the Institute of Medicine. J Am Med Assoc. 2015;313(21):2121-2.

3. Associate Minister of Health. Healthy ageing strategy. Wellington: Ministry of Health; 2016. https://www.health.govt.nz/system/files/documents/ publications/healthy-ageing-strategy_june_2017.pdf, Accessed 2 June 2018

4. Deloitte. Dementia economic impact report 2016: Alzheimers New Zealand; 2017. http://www.alzheimers.org.nz/getmedia/79f7fd09-93fe43b0-a837-771027bb23c0/Economic-Impacts-of-Dementia-2017.pdf/, Accessed 28 Sept 2018.

5. Luppa M, Luck T, Weyerer S, Konig HH, Brahler E, Riedel-Heller SG. Prediction of institutionalization in the elderly. A systematic review. Age Ageing. 2010;39(1):31-8.

6. Winblad B, Amouyel P, Andrieu S, Ballard C, Brayne C, Brodaty H, et al. Defeating Alzheimer's disease and other dementias: a priority for European science and society. Lancet Neurol. 2016;15(5):455-532.

7. Horr T, Messinger-Rapport B, Pillai JA. Systematic review of strengths and limitations of randomized controlled trials for non-pharmacological interventions in mild cognitive impairment: focus on Alzheimer's disease. J Nutr Health Aging. 2015;19(2):141-53.
8. Mahoney-Sanchez L, Belaidi AA, Bush Al, Ayton S. The complex role of apolipoprotein $\mathrm{E}$ in Alzheimer's disease: an overview and update. J Mol Neurosci. 2016;60(3):325-35.

9. Sun J-H, Tan L, Wang H-F, Tan M-S, Tan L, Li J-Q, et al. Genetics of vascular dementia: systematic review and meta-analysis. J Alzheimers Dis. 2015;46(3): 611-29.

10. Wisdom NM, Callahan JL, Hawkins KA. The effects of apolipoprotein E on non-impaired cognitive functioning: a meta-analysis. Neurobiol Aging. 2011; 32(1):63-74.

11. Norton S, Matthews FE, Barnes DE, Yaffe K, Brayne C. Potential for primary prevention of Alzheimer's disease: an analysis of population-based data. Lancet Neurol. 2014;13(8):788-94.

12. Frisardi V, Solfrizzi V, Seripa D, Capurso C, Santamato A, Sancarlo D, et al. Metabolic-cognitive syndrome: a cross-talk between metabolic syndrome and Alzheimer's disease. Ageing Res Rev. 2010:9(4):399-417.

13. Cooper C, Sommerlad A, Lyketsos CG, Livingston G. Modifiable predictors of dementia in mild cognitive impairment: a systematic review and metaanalysis. Am J Psychiat. 2015;172(4):323-34.

14. Wu L, Sun D, Tan Y. Intake of fruit and vegetables and the incident risk of cognitive disorders: a systematic review and meta-analysis of cohort studies. J Nutr Health Aging. 2017;21(10):1284-90.

15. Solfrizzi V, Custodero C, Lozupone M, Imbimbo BP, Valiani V, Agosti P, et al. Relationships of dietary patterns, foods, and micro- and macronutrients with Alzheimer's disease and late-life cognitive disorders: a systematic review. J Alzheimers Dis. 2017:59(3):815-49.

16. Loughrey DG, Lavecchia S, Brennan S, Lawlor BA, Kelly ME. The impact of the Mediterranean diet on the cognitive functioning of healthy older adults: a systematic review and meta-analysis. Adv Nutr. 2017;8(4):571-86.

17. Crichton GE, Bryan J, Murphy KJ. Dietary antioxidants, cognitive function and dementia - a systematic review. Plant Foods Hum Nutr. 2013:68(3):279-92.

18. Cao L, Tan L, Wang HF, Jiang T, Zhu XC, Lu H, et al. Dietary patterns and risk of dementia: a systematic review and meta-analysis of cohort studies. Mol Neurobiol. 2016;53(9):6144-54.

19. Francis $H$, Stevenson $\mathrm{R}$. The longer-term impacts of Western diet on human cognition and the brain. Appetite. 2013;63:119-28.

20. Hu FB. Dietary pattern analysis: a new direction in nutritional epidemiology. Curr Opin Lipidol. 2002;13(1):3-9.

21. Milte CM, McNaughton SA. Dietary patterns and successful ageing: a systematic review. Eur J Nutr. 2016;55(2):423-50.

22. Robberecht $H$, De Bruyne $T$, Hermans $N$. Effect of various diets on biomarkers of the metabolic syndrome. Int J Food Sci Nutr. 2017;68(5):627-41.

23. Newby PK, Tucker KL. Empirically derived eating patterns using factor or cluster analysis: a review. Nutr Rev. 2004;62(5):177-203.

24. Samieri C, Jutand MA, Feart C, Capuron L, Letenneur L, Barberger-Gateau P. Dietary patterns derived by hybrid clustering method in older people: association with cognition, mood, and self-rated health. J Am Diet Assoc. 2008;108(9):1461-71.

25. Parrott MD, Shatenstein B, Ferland G, Payette $H$, Morais JA, Belleville $S$, et al. Relationship between diet quality and cognition depends on socioeconomic position in healthy older adults. J Nutr. 2013;143(11):1767-73.

26. Kesse-Guyot E, Andreeva VA, Jeandel C, Ferry M, Hercberg S, Galan P. A healthy dietary pattern at midlife is associated with subsequent cognitive performance. J Nutr. 2012;142(5):909-15.

27. Kesse-Guyot E, Andreeva VA, Ducros V, Jeandel C, Julia C, Hercberg S, et al. Carotenoid-rich dietary patterns during midlife and subsequent cognitive function. Br J Nutr. 2014;111(5):915-23.

28. Okubo $H$, Inagaki $H$, Gondo $Y$, Kamide $K$, Ikebe $K$, Masui $Y$, et al. Association between dietary patterns and cognitive function among 70-year-old Japanese elderly: a cross-sectional analysis of the SONIC study. Nutr J. 2017;16:56.

29. Chan R, Chan D, Woo J. A cross sectional study to examine the association between dietary patterns and cognitive impairment in older Chinese people in Hong Kong. J Nutr Health Aging. 2013;17(9):757-65.

30. Corley J, Starr JM, MCNeill G, Deary IJ. Do dietary patterns influence cognitive function in old age? Int Psychogeriatr. 2013;25(9):1393-407.

31. Granic A, Davies K, Adamson A, Kirkwood T, Hill TR, Siervo M, et al. Dietary patterns high in red meat, potato, gravy, and butter are associated with poor cognitive functioning but not with rate of cognitive decline in very old adults. J Nutr. 2016;146(2):265-74.

32. Akbaraly TN, Singh-Manoux A, Marmot MG, Brunner EJ. Education attenuates the association between dietary patterns and cognition. Dement Geriatr Cogn Disord. 2009;27(2):147-54. 
33. Gardener SL, Rainey-Smith SR, Barnes MB, Sohrabi HR, Weinborn M, Lim YY, et al. Dietary patterns and cognitive decline in an Australian study of ageing. Mol Psychiatry. 2015;20(7):860-6.

34. Gu YA, Nieves JW, Stern Y, Luchsinger JA, Scarmeas N. Food combination and Alzheimer disease risk. A protective diet. Arch Neurol. 2010;67(6):699-706.

35. Berendsen AM, Kang JH, Feskens EJM, de Groot CPGM, Grodstein F, Van de Rest $O$. Association of long-term adherence to the mind diet with cognitive function and cognitive decline in American women. J Nutr Health Aging. 2018;22(2):222-9.

36. Torres SJ, Lautenschlager NT, Wattanapenpaiboon N, Greenop KR, Beer C, Flicker $L$, et al. Dietary patterns are associated with cognition among older people with mild cognitive impairment. Nutrients. 2012;4(11):1542-51.

37. Thompson JMD, Wall C, Becroft DMO, Robinson E, Wild CJ, Mitchell EA. Maternal dietary patterns in pregnancy and the association with small-forgestational-age infants. Br J Nutr. 2010;103(11):1665-73.

38. Wall CR, Gammon CS, Bandara DK, Grant CC, Carr PEA, Morton SMB. Dietary patterns in pregnancy in New Zealand - influence of maternal sociodemographic, health and lifestyle factors. Nutrients. 2016;8(5):16.

39. Beck KL, Kruger R, Conlon CA, Heath A-LM, Matthys C, Coad J, et al. Suboptimal iron status and associated dietary patterns and practices in premenopausal women living in Auckland, New Zealand. Eur J Nutr. 2013; 52(2):467-76.

40. Schrijvers JK, McNaughton SA, Beck KL, Kruger R. Exploring the dietary patterns of young New Zealand women and associations with BMI and body fat. Nutrients. 2016;8(8):15.

41. Beck KL, Jones B, Ullah I, McNaughton SA, Haslett SJ, Stonehouse W. Associations between dietary patterns, socio-demographic factors and anthropometric measurements in adult new Zealanders: an analysis of data from the 2008/09 New Zealand adult nutrition survey. Eur J Nutr 2018:57(4):1421-33.

42. Saeedi P, Black K, Haszard J, Skeaff S, Stoner L, Davidson B, et al. Dietary patterns, cardiorespiratory and muscular fitness in 9-11-year-old children from Dunedin, New Zealand. Nutrients. 2018;10(7):887.

43. Craig C, Marshall A, Sjöström M, Bauman A, Booth M, Ainsworth B, et al International physical activity questionnaire: 12-country reliability and validity. Med Sci Sports Exerc. 2003;35(8):1381-95.

44. Marfell-Jones M, Stewart A, De Ridder J. International standards for anthropometric assessment. Wellington: International Society for the Advancement of Kinanthropometry; 2012.

45. F. Hoffmann-La Roche Ltd: cobas b 101 system. https://diagnostics.roche. com/global/en/products/instruments/cobas-b-101.html 2018. Accessed 28 Sept 2018.

46. Beck KL, Kruger R, Conlon CA, Heath A-LM, Coad J, Matthys C, et al. The relative validity and reproducibility of an iron food frequency questionnaire for identifying iron-related dietary patterns in young women. J Acad Nutr Diet. 2012;112(8):1177-87.

47. Nasreddine ZS, Phillips NA, Bedirian V, Charbonneau S, Whitehead V, Collin I, et al. The Montreal cognitive assessment, MoCA: a brief screening tool for mild cognitive impairment. J Am Geriatr Soc. 2005;53(4):695-9.

48. Massey University: The REACH Study. http://www.massey.ac.nz/reachstudy 2018. Accessed 31 May 2018

49. Exeter DJ, Zhao J, Crengle S, Lee A, Browne M. The New Zealand indices of multiple deprivation (IMD): a new suite of indicators for social and health research in Aotearoa, New Zealand. PLoS One. 2017;12:8.

50. Beck KL, Houston ZL, McNaughton SA, Kruger R. Development and evaluation of a food frequency questionnaire to assess nutrient intakes of adult women in New Zealand. Nutr Diet. 2018. https://doi.org/10.1111/1747-0080.12472.

51. Houston ZL. Development and validation of a semi-quantitative food frequency questionnaire to assess dietary intake of adult women living in New Zealand: a thesis presented in partial fulfillment of the requirements for the degree of masters of science in nutrition and dietetics, Massey University, Albany, New Zealand [thesis]: Massey University; 2014.

52. New Zealand food composition database 2017. New Zealand food composition database: New Zealand FOODfiles ${ }^{(\mathrm{Tm})} 2016$ version 01. https:// www.foodcomposition.co.nz/foodfiles. Accessed 25 Sept 2018.

53. Goldberg GR, Black AE, Jebb SA, Cole TJ, Murgatroyd PR, Coward WA, et al. Critical-evaluation of energy intake data using fundamental principles of energy physiology: 1. Derivation of cut-off limits to identifying underrecording. Eur J Clin Nutr. 1991;45(12):569-81.

54. Nelson M, Atkinson M, Meyer J. A photographic atlas of food portion sizes: MAFF Publications; 1997
55. Stonehouse W, Conlon CA, Podd J, Hill SR, Minihane AM, Haskell C, et al. DHA supplementation improved both memory and reaction time in healthy young adults: a randomized controlled trial. Am J Clin Nutr. 2013;97(5):1134-43

56. Kennedy DO, Wightman EL, Forster J, Khan J, Haskell-Ramsay CF, Jackson PA. Cognitive and mood effects of a nutrient enriched breakfast bar in healthy adults: a randomised, double-blind, placebo-controlled, parallel groups study. Nutrients. 2017;9(12):21.

57. Grundy SM, Cleeman JI, Daniels SR, Donato KA, Eckel RH, Franklin BA, et al. Diagnosis and management of the metabolic syndrome - an American Heart Association/National Heart, Lung, and Blood Institute scientific statement. Circulation. 2005;112(17):2735-52.

58. R Core Team. R: a language and environment for statistical computing. Vienna: R Foundation for statistical Computing; 2016.

59. Field AP. Discovering statistics using IBM SPSS statistics. 5th ed. Los Angeles: Sage; 2018.

60. Bland JM, Altman DG. Statistical methods for assessing agreement between two methods of clinical measurement. Int J Nurs Stud. 2010;47(8):931-6.

61. Warton DI. Penalized normal likelihood and ridge regularization of correlation and covariance matrices. J Am Stat Assoc. 2008;103(481):340-9.

\section{Ready to submit your research? Choose BMC and benefit from:}

- fast, convenient online submission

- thorough peer review by experienced researchers in your field

- rapid publication on acceptance

- support for research data, including large and complex data types

- gold Open Access which fosters wider collaboration and increased citations

- maximum visibility for your research: over $100 \mathrm{M}$ website views per year

At BMC, research is always in progress.

Learn more biomedcentral.com/submissions 